\title{
ON BOUNDARY VALUES OF HOLOMORPHIC FUNCTIONS ON BALLS
}

\author{
JOSIP GLOBEVNIK
}

\begin{abstract}
It is a result of Agranovski and Valski for which Nagel and Rudin, and Stout have given alternate proofs, that if $B$ is the open unit ball in $\mathbf{C}^{n}$ and if $f \in C(\partial B)$ has the property that for every complex line $\Lambda \subset \mathbf{C}^{n}, f \mid(\Lambda \cap \partial B)$ has a continuous extension to $\Lambda \cap \bar{B}$ which is holomorphic in $\Lambda \cap B$, then $f$ has a continuous extension to $\bar{B}$ which is holomorphic in $B$. In the paper we give an easier, more geometric proof of this result and then prove the local version of this result.
\end{abstract}

The following proposition is a result of Agranovski and Valski [1].

Proposition 1. Let $B$ be the open unit ball in $\mathbf{C}^{n}$ and let $f \in C(\partial B)$ have the property that for every complex line $\Lambda \subset \mathbf{C}^{n}, f \mid(\Lambda \cap \partial B)$ has a continuous extension to $\Lambda \cap \bar{B}$ which is holomorphic in $\Lambda \cap B$. Then $f$ has a continuous extension to $\bar{B}$ which is holomorphic in $B$.

Three different proofs of this result are known. Agranovski and Valski use the theorem of Severi [7] and need the existence of Haar measure on unitary group. Nagel and Rudin [2] obtain this result as a consequence of their characterization of Moebius-invariant subspaces of $C(\partial B)$. Stout [5] proves the result for general smoothly bounded domains in $\mathbf{C}^{n}$ - he shows first that every $f$ satisfying the conditions of Proposition 1 is a weak solution of tangential Cauchy-Riemann equations and then applies a theorem of Weinstock [8]. In [6], Stout proves a local version of the main result of [5].

In this note we present a new, more geometric proof of Proposition 1 (we hope that this will contribute to a better intuitive understanding of Proposition 1) and then use the main argument of the proof to prove a local version of Proposition 1. Our proof uses the well-known fact that the boundary values of functions from the bidisc algebra are those continuous functions $g$ on the torus whose Fourier coefficients

$$
\iint e^{i j \theta} e^{i k \varphi} g\left(e^{i \theta}, e^{i \varphi}\right) d \theta d \varphi
$$

vanish whenever at least one of the indices $j, k$ is positive [3].

Proof of Proposition 1. It is easy to see that if $\tilde{f}$ is an extension of $f$ to the closed ball $\bar{B}$ such that for every complex line $\Lambda, \tilde{f} \mid(\Lambda \cap \bar{B})$ is continuous and $\tilde{f} \mid(\Lambda \cap B)$ is holomorphic, then $\tilde{f}$ is continuous on $\bar{B}$ and holomorphic on $B$.

Received by the editors August 15, 1981.

1980 Mathematics Subject Classification. Primary 32A40.

() 1982 American Mathematical Society 0002-9939/81/0000-11 16/\$02.00 
Consequently, to prove Proposition 1 it is enough to prove that if $p \in B$ and if $\Lambda_{1}, \Lambda_{2}$ are two complex lines passing through $p$ then the holomorphic extensions of $f\left|\left(\Lambda_{1} \cap \partial B\right), f\right|\left(\Lambda_{2} \cap \partial B\right)$ into $\Lambda_{1} \cap B, \Lambda_{2} \cap B$, respectively, have the same value at $p$. Since Moebius transformations map $\bar{B}$ homeomorphically onto $\bar{B}, B$ biholomorphically onto $B$, and complex lines to complex lines $[2,4]$ it is enough to prove this for $p=0$.

So let $x, y \in \partial B$, let $\Delta \subset \mathbf{C}$ be the open unit disc, and let $f_{x}, f_{y}$ be the functions in the disc algebra that satisfy $f_{x}(s)=f(s x), f_{y}(s)=f(s y)(s \in \partial \Delta)$. We have to prove that $f_{x}(0)=f_{y}(0)$. We will do this by proving that $f_{x}$ and $f_{y}$ are two slice functions of a function $\tilde{g}$ from the bidisc algebra. We first construct a torus $T$ contained in $\partial B$ which contains the circles $L_{x}=\{s x: s \in \partial \Delta\}$ and $L_{y}=\{s y: s \in \partial \Delta\}$. Choose $\tau \in \partial \Delta$ such that $\langle\tau x \mid y\rangle$ is real and put $u=(\tau x+y) / 2, w=(\tau x-y) / 2$. Then $|u|^{2}+\mid w$ $1^{2}=1$, and since $\langle\tau x \mid y\rangle$ is real we have $\langle u \mid w\rangle=0$. Consequently the torus $T=\{s u+t w: s, t \in \partial \Delta\}$ is contained in $\partial B$. Note also-we will need this only in the proof of Proposition 2-that if $\varepsilon>0$ and if $L_{y} \subset D=\{z \in$ $\left.\partial B: \operatorname{dist}\left(z, L_{x}\right)<\varepsilon\right\}$ then $T \subset D$. Define $g(s, t)=f(s u+t w)(s, t \in \partial \Delta)$. By the assumption for each $s$ the function $t \mapsto f(s u+t w)$ extends to a function in the disc algebra. Consequently

$$
\iint e^{i j \theta} e^{i k \varphi} g\left(e^{i \theta}, e^{i \varphi}\right) d \theta d \varphi=0
$$

whenever at least one of the indices $j, k$ is positive which implies that $g$ extends to a function $\tilde{g}$ in the bidisc algebra. Since

$$
\left.\begin{array}{l}
f_{x}(t)=f\left(t \tau^{-1}(u+w)\right)=\tilde{g}\left(t\left(\tau^{-1}, \tau^{-1}\right)\right), \\
f_{y}(t)=f(t(u-w))=\tilde{g}(t(1,-1))
\end{array}\right\} \quad(t \in \partial \Delta)
$$

it follows that $f_{x}(0)=\tilde{g}(0,0)=f_{y}(0)$. This completes the proof.

We use the same idea to prove the following local version of Proposition 1 (see also [4, Theorem 18.1.12] and [6, Theorem II.1]).

Proposition 2. Let $B \subset \mathbf{C}^{n}, n>1$, be the open unit ball, let $x_{0} \in \partial B$ and suppose that $t<1$. Let

$$
\Gamma=\left\{x \in \partial B: t<\operatorname{Re}\left\langle x \mid x_{0}\right\rangle\right\}, \quad \Omega=\left\{x \in B: t<\operatorname{Re}\left\langle x \mid x_{0}\right\rangle\right\} .
$$

Suppose that $f \in C(\Gamma)$ has the property that for every complex line $\Lambda$ such that $\Lambda \cap \Gamma$ is a circle (i.e. such that $\Lambda \cap \Gamma=\Lambda \cap \partial B), f \mid(\Lambda \cap \partial B)$ has a continuous extension to $\Lambda \cap \bar{B}$ which is holomorphic in $\Lambda \cap B$. Then f has a continuous extension to $\Gamma \cup \Omega$ which is holomorphic on $\Omega$.

Lemma 1. Let $\Gamma, \Omega$ and $f$ be as in Proposition 2. Let $\mathrm{L}$ be the set of all complex lines $\Lambda$ such that $\Lambda \cap \Gamma$ is a circle. Let $x \in \Omega$ and let $y \neq 0$ be such that $\Lambda=\{x+\zeta y: \zeta \in$ C) $\in \mathcal{L}$. There is an $\varepsilon>0$ such that whenever $|z-y|<\varepsilon$ then $\Lambda_{z}=\{x+\zeta z: \zeta \in \mathbf{C}\}$ $\in \mathfrak{L}$ and the holomorphic extensions of $f|(\Lambda \cap \partial B), f|\left(\Lambda_{z} \cap \partial B\right)$ into $\Lambda \cap B$, $\Lambda_{z} \cap B$, respectively, have the same value at $x$. 
Proof. Let $\phi$ be the Moebius transformation mapping 0 to $x$. Let $S \subset \partial B$ be a neighbourhood of $L=\phi^{-1}(\Lambda \cap \partial B)$ such that $\phi(S) \subset \Gamma$. If $v \in \partial B$ is such that $L^{\prime}=\{t v: t \in \partial \Delta\}$ is sufficiently close to $L$ then $L$ and $L^{\prime}$ are contained in a torus $T \subset S$ (see the proof of Proposition 1). It follows that there is an $\varepsilon>0$ such that if $|z-y|<\varepsilon$ then $\Lambda_{z} \in \mathcal{L}$ and $L$ and $\phi^{-1}\left(\Lambda_{z} \cap \partial B\right)$ are contained in a torus $T \subset \partial B$ such that $\phi(T) \subset \Gamma$. Consequently $\phi$ maps the complex circles (=intersections of $\partial B$ with complex lines) contained in $T$ to complex circles contained in $\Gamma$. By the argument used in the proof of Proposition 1 it follows that the holomorphic extensions of $f|(\Lambda \cap \partial B), f|\left(\Lambda_{z} \cap \partial B\right)$ to $\Lambda \cap B, \Lambda_{z} \cap B$, respectively, have the same value at $x$. This completes the proof.

Lemma 2. Let $g$ be a bounded function on $B$ and let $x_{1}, x_{2}, \ldots, x_{n}$ span $\mathbf{C}^{n}$. Suppose that $g \mid(\Lambda \cap B)$ is holomorphic for every complex line $\Lambda$ of the form $\Lambda=\left\{p+\zeta x_{i}: \zeta\right.$ $\in \mathbf{C}\}, p \in B, 1 \leqslant i \leqslant n$. Then $g$ is holomorphic on $B$.

Proof. If $L: \mathbf{C}^{n} \rightarrow \mathbf{C}^{n}$ is the linear map which maps the $i$ th coordinate vector to $x_{i}, 1 \leqslant i \leqslant n$, then $g \circ L$ is bounded on $L^{-1}(B)$ and analytic in each variable separately. Consequently $g \circ L$ is analytic on $L^{-1}(B)$ and so is $g$ on $B$.

Proof of Proposition 2. Let $\mathcal{L}$ be as in Lemma 1. Let $x \in \Omega$ and let $y_{0}, y_{1} \in \partial B$ be such that $\Lambda_{0}=\left\{x+\zeta y_{0}: \zeta \in \mathbf{C}\right\}, \Lambda_{1}=\left\{x+\zeta y_{1}: \zeta \in \mathbf{C}\right\}$ both belong to $\mathcal{L}$. It is easy to construct a path $y:[0,1] \rightarrow \partial B, y(0)=y_{0}, y(1)=y_{1}$, such that $\{x+\zeta y(t): \zeta$ $\in \mathbf{C}\} \in \mathcal{L}(0 \leqslant t \leqslant 1)$. By Lemma 1 it follows that the holomorphic extensions of $f\left|\left(\Lambda_{0} \cap \partial B\right), f\right|\left(\Lambda_{1} \cap \partial B\right)$ into $\Lambda_{0} \cap B, \Lambda_{1} \cap B$, respectively, have the same value at $x$. Since every $x \in \Omega$ belongs to some $\Lambda \in \mathcal{L}$ it follows that $f$ extends to a function $\tilde{f}$ on $\Omega \cap \Gamma$ such that for each $\Lambda \in \mathcal{E}, \tilde{f} \mid(\Lambda \cap B)$ coincides with the holomorphic extension of $f \mid(\Lambda \cap \partial B)$ into $\Lambda \cap B$.

Let $x \in \Omega$. Applying the maximum modulus theorem and using the continuity of $f$ we see that there are an open ball $U \subset \Omega$, centered at $x$, and a nonempty open set $W \subset \mathbf{C}^{n}$ such that $\tilde{f} \mid U$ is bounded and such that $\tilde{f} \mid(U \cap \Lambda)$ is holomorphic whenever $\Lambda=\{y+\zeta p: \zeta \in \mathbf{C}\}, y \in U, p \in W$. By Lemma 2 it follows that $\tilde{f}$ is analytic on $U$. This proves that $\tilde{f}$ is analytic in $\Omega$. To prove the continuity of $\tilde{f}$ at $x \in \Gamma$, fix $x \in \Gamma$, let $\varepsilon>0$ and choose $\tau<1$ so close to 1 that $\Gamma_{\tau}=\{y \in \partial B: \tau<$ $\operatorname{Re}\langle y \mid x\rangle\} \subset \Gamma$ and that $|f(y)-f(x)|<\varepsilon\left(y \in \Gamma_{\tau}\right)$. Let $z \in \Omega_{\tau}=\{y \in B: \tau<$ $\operatorname{Re}\langle y \mid x\rangle\}$. Then $z$ belongs to a complex line $\Lambda$ such that $\Lambda \cap \partial B \subset \Gamma_{\tau}$. We have $|f(p)-f(x)|<\varepsilon(p \in \Lambda \cap \partial B)$ and by the maximum modulus theorem it follows that $|f(z)-f(x)|<\varepsilon$. This completes the proof.

The author is indebted to Joseph Cima with whom he had some very stimulating discussions on this subject.

This work was supported in part by the Boris Kidrič Fund, Ljubljana, Yugoslavia.

\section{REFERENCES}

1. M. L. Agranovski and R. E. Valski, Maximality of invariant algebras of functions, Siberian Math. J. 12 (1971), 1-7.

2. A. Nagel and W. Rudin, Moebius-invariant function spaces on balls and spheres, Duke Math. J. 43 (1976), 841-865.

3. W. Rudin, Function theory in polydiscs, Benjamin, New York, 1969. 
4. _ Function theory in the unit ball of $\mathbf{C}^{\prime \prime}$, Die Grundlehren der Math. Wissenschaften, vol. 241. Springer-Verlag, New York and Berlin, 1980.

5. E. L. Stout, The boundary values of holomorphic functions of several complex variables, Duke Math. J. 44 (1977), 105-108.

6. Analytic continuation and boundary continuity of functions of several complex variables, Proc. Roy. Soc. Edinburgh Sect. 89A (1981), 63-74.

7. B. V. Śabat, Introduction to complex analysis, "Nauka", Moscow, 1969.

8. B. M. Weinstock. An approximation theorem for $\bar{\partial}$-closed forms of type $(n, n-1)$, Proc. Amer. Math. Soc. 26 (1970), 625-628.

Institute of Mathematics, Physics and Mechanics, E. K. University of Ljubljana, Ljubljana, Yugoslavia 